Archives

4| 1989

Varia

\title{
De l'archéologie à l'histoire : le cas de la Basilicate italique
}

\section{Angelo Bottini}

\section{(C) OpenEdition}

\section{Journals}

Édition électronique

URL : http://journals.openedition.org/ccrh/2912

DOI : $10.4000 /$ ccrh.2912

ISSN : 1760-7906

Éditeur

Centre de recherches historiques - EHESS

Édition imprimée

Date de publication : 15 octobre 1989

ISSN : 0990-9141

Référence électronique

Angelo Bottini, « De l'archéologie à l'histoire : le cas de la Basilicate italique », Les Cahiers du Centre de Recherches Historiques [En ligne], 4 | 1989, mis en ligne le 13 avril 2009, consulté le 19 avril 2019. URL: http://journals.openedition.org/ccrh/2912; DOI : 10.4000/ccrh.2912

Ce document a été généré automatiquement le 19 avril 2019

Article L.111-1 du Code de la propriété intellectuelle. 


\title{
De l'archéologie à l'histoire : le cas de la Basilicate italique
}

\author{
Angelo Bottini
}

1 Dans son livre The Western Greeks, T.J. Dunbabin commentait par ces mots les quelques pages qu'il y consacrait à Métaponte. Il résumait ainsi notre connaissance du monde indigène de la Basilicate et des rapports que ce monde entretenait avec les colons grecs installés sur les côtes de la région.

2 Aujourd'hui encore, rien, dans ces propos, ne peut être tenu pour complètement faux. Mais un quart de siècle de recherches historiques (qui ont débuté avec la création de la «Surintendance archéologique » de Basilicate, en 1964) nous permet désormais, comme on va le voir, d'esquisser une histoire de ces régions à l'époque antique tout à fait différente de celle que retenaient les chercheurs de la génération précédente - surtout pour la période comprise entre le début de la colonisation grecque et l'entrée dans la sphère d'influence romaine, c'est-à-dire entre le viII ${ }^{\mathrm{e}}$ et le $\mathrm{III}^{\mathrm{e}}$ siècle avant J.-C.

3 Jetons d'abord un coup d'oeil à la géographie. La Basilicate d'aujourd'hui, c'est l'antique Lucanie, à l'exception de presque toute sa bande côtière le long de la Mer tyrrhénienne, qui est actuellement exclue de la Basilicate et fait partie de la Campanie. Elle se présente comme une région avant tout montagneuse, où l'on ne trouve qu'une seule vraie plaine, sur les côtes de la Mer Ionienne. D'autre part, sur le versant oriental, sa bordure de collines correspond à des bandes de territoire apulien (de Daunie ou de Peucétie) limitées en extension, mais historiquement très importantes, et qui ont été rattachées à la Basilicate après l'époque classique. 


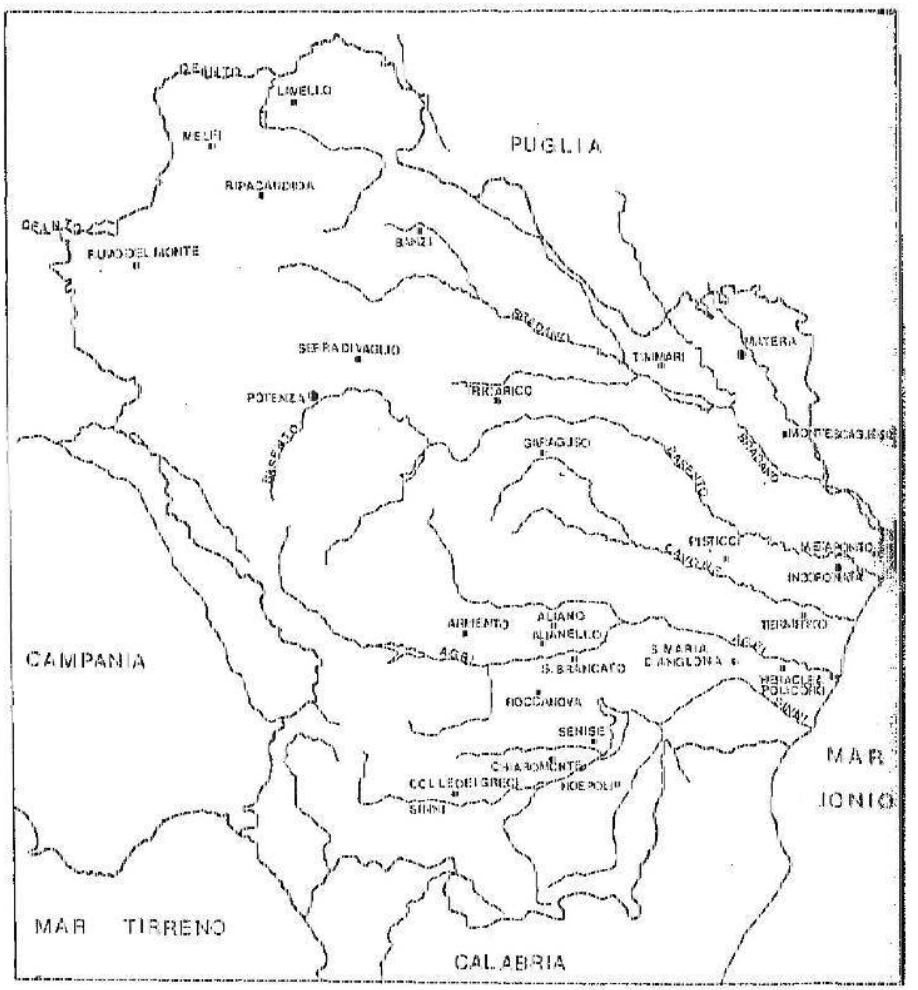

Principaux sites antiques de la Basilicate (du VIII au III siècle av. J.-C.) roumaine auquel on doit la naissance même de la recherche archéologique dans cette partie de la Grande Grèce, une des caractéristiques importantes de la Lucanie est d'être traversée par de nombreux cours d'eau. Cinq d'entre eux (du Sud vers le Nord-Est : le Sinni, l'Agri, le Carone, le Basento, enfin le Bradano) coulent vers la Mer ionienne. Le sixième, l'Ofanto ("l'Aufide, dont le cours borde les royaumes de Daunus l'Apulien », comme dit Horace dans ses Odes 5, 14,25), qui lui aussi prend sa source dans les montagnes de l'Apennin, se dirige au contraire vers l'Adriatique. du Premier Age du Fer, en gros au cours du dernier quart du VIII ${ }^{\mathrm{e}}$ siècle av. J.-C. A ce sujet, les recherches les plus récentes ont surtout mis en lumière les deux traits suivants : d'une part, de fortes différences de densité entre les diverses zones ; d'autre part, des clivages culturels également nets, mais qui ne coïncident pas avec ces différences.

Les territoires de l'intérieur, dans leur ensemble, apparaissent comme à moitié déserts. On y rencontre les traces de petits établissements de dimensions modestes, qui indiquent un niveau d'évolution encore très bas. Néanmoins, les pratiques funéraires autorisent à y reconnaître déjà des clivages sociaux qu'il semble légitime de tenir aussi pour ethniques - clivages destinés à se révéler pleinement au cours de la phase suivante, la phase archaïque.

Les peuples qui occupent la partie montagneuse à la limite du Bruttium et les vallées de la bordure méridionale de la région, les vallées des fleuves Agri et Sinni (dans l'Antiquité, l' Akinis et le Siris), appartiennent à ce que l'archéologie du début du siècle appelait la "Fossakultur », la " culture des tombes à fosse », caractéristique d'une large portion des côtes tyrrhéniennes. Ils pratiquent en effet le rite de l'inhumation en position allongée 
sur le dos, et justement dans des fosses d'un type très simple. Dans ces indigènes-là, il faut, selon toute probabilité, reconnaître les Oenôtres, un des peuples italiotes les plus connus de la tradition historiographique grecque.

8 Les habitats du centre de la région de Potenza et des territoires de l'extrémité orientale de la Basilicate doivent au contraire être attribués à des groupes proches des Iapyges des régions voisines, en Apulie, qui pratiquaient l'inhumation en position recroquevillée et parfois dans des tombes à tumulus.

Des usages tout à fait analogues se manifestent aussi chez les habitants de la bande côtière du golfe de Tarente, qui occupaient en particulier les deux villages protohistoriques les plus peuplés que l'on connaisse jusqu'ici, tous deux situés au voisinage de sites occupés par la suite par les colons grecs. Il s'agit de San TeodoroIncoronata, Métaponte et de Santa Maria d'Anglona - Valle Sorrigliano, en entrant dans l'arrière-pays de Siris-Polieion. Ce dernier est peut-être à identifier avec l'un des principaux sites des Chônes. Les sources dont s'inspire Strabon $(\mathrm{VI}, 1,14=\mathrm{C} 264)$ nous montrent en effet les Chônes installés à l'endroit même où s'élèvera par la suite la nouvelle cité.

10 Chose importante, la découverte d'objets d'importation (de la céramique, des colliers) prouve que ces groupes tournés vers la Mer ionienne constituent le point de référence des navigateurs orientaux dès l'époque qui précède le moment des apoikiai, des fondations coloniales.

$11 \mathrm{Au}$ point de vue socio-économique, ces communautés se révèlent encore très peu structurées. De l'analyse des nécropoles, il semble en effet possible de saisir, comme ailleurs en Italie, une société à base égalitaire, dans laquelle n'émergent que des figures individuelles de "chefs", ensevelis avec un mobilier spécifique, parfois à côté de leur compagne. Les contacts avec les étrangers étaient probablement l'apanage de ces chefs, dont l'épée constituait l'élément distinctif.

12 Au tout début du vII ${ }^{\mathrm{e}}$ siècle av. J.-C., la présence grecque tend à se stabiliser. Peu après, entre les bouches de l'Agri et celles du Sinni, est en effet fondée Siris, colonie de la cité ionienne de Colophon (située sur la côte anatolienne). Les fouilles les plus récentes qui aient eu lieu à l'intérieur de l'actuelle petite ville de Policoro ont enfin permis de retrouver quelques restes des maisons de l'établissement du courant du viI ${ }^{\mathrm{e}}$ siècle. Mais Siris (située, à ce qu'il semble, à l'emplacement précis de la future Heracleia, qui héritera même de ses lieux sacrés) reste cependant surtout connue par ses sépultures, que la pratique de l'incinération, complètement ignorée jusqu'alors, permet d'identifier facilement. Face à ces événements, quel est le sort des peuples indigènes ?

Sans oublier que l'attitude des Grecs à l'égard des indigènes, loin de suivre des règles constantes, se traduit par une gamme plutôt étendue de comportements, il faut établir une distinction, qui correspond en gros aux conséquences d'une situation géographique différente. Car le fait que les Grecs s'intéressent avant tout au contrôle complet de la plaine côtière détermine une pression sur les villages italiques qui va jusqu'à en provoquer soit la disparition (à titre de contre-épreuve, on signalera que des inhumations individuelles d'indigènes apparaissent à l'intérieur même des nécropoles urbaines de Siris ), soit la complète acculturation. Dans le cas du site de l'Incoronata, si l'on accepte l'hypothèse qu'il y a eu là, dans ce centre antérieur à Métaponte, coexistence des deux communautés, c'est l'acculturation qui pourrait s'être produite. 
14 La situation du lointain arrière-pays des Oenôtres est complètement différente. La consolidation de la présence coloniale grecque exerce sur eux des effets tout à fait positifs, pour des motifs qui méritent une attention particulière.

Le plus évident de ces effets est une croissance démographique progressive, attestée par les nombreuses nécropoles, avec des tombes riches en vaisselle de la "série principale » de la production géométrique et subgéométrique bichrôme, dont la syntaxe décorative se rattache directement à celle qui était en usage au voisinage de la côte ionienne dans la dernière partie du viII ${ }^{e}$ siècle.

Parallèlement, il faut noter l'apparition soudaine d'indices d'un processus de structuration sociale (et c'est là le phénomène le plus impressionnant). Il est attesté en premier lieu par les fouilles de la très vaste nécropole d'Alianello d'Aliano (dans la moyenne vallée de l'Agri). Parmi beaucoup d'autres sépultures, on y a découvert, dans des tombes remarquables aussi par leur aspect extérieur, les sépultures d'un groupe d'individus de condition nettement privilégiée, comme c'est surtout évident dans le cas des femmes.

17 Pour les hommes, en effet, il apparaît longtemps de règle d'observer la sobriété traditionnelle, et de se borner à montrer leur rôle guerrier par le moyen de leur grande épée. Dans le cas des femmes, au contraire, l'adoption des coutumes paraît rapide. Aux grandes et complexes parures ornementales, riches en colliers et en appliques pour vêtements, faites de métaux variés ainsi que d'os et d'ambre (s'agit-il des vêtements de noces?), parures qui font penser aux chalkochitones homériques, on trouve associés, en effet, les biens de prestige importés.

Qu'est-ce qui a incité les nouveaux venus à s'intéresser aussi rapidement aux territoires de l'intérieur? A cette question, on répond d'habitude en évoquant le mécanisme colonial bien connu de l'échange des matières premières et de la main-d'oeuvre contre des objets fabriqués et des produits agricoles de prix, encore inconnus du monde indigène, comme le vin et l'huile. Cette explication est sans aucun doute vraie, mais, au moins dans le cas présent, elle ne semble pas épuiser toutes les possibilités. Dans ces tombes, à côté de céramique corinthienne ou de type corinthien mais de fabrication coloniale (parmi les plus anciennes, signalons un aryballe ovoïde du protocorinthien moyen et une coupe du type Thapsos sans panneau), figurent en effet des objets de bronze (chaudrons, bassins et phiales, travaillés au repoussé) qu'il faut plutôt attribuer à la métallurgie étrusque ou étrusco-campanienne.

19 Il est donc évident que les Oenôtres de ces grandes vallées ne sont pas (ou ne sont pas toujours) les ultimes destinataires des relations ainsi nouées. Ils n'ont joué souvent que le rôle - précieux - d'intermédiaires, le long d'un itinéraire d'échanges par voie de terre entre le pôle grec sur la Mer ionienne et le pôle étrusco-campanien sur le Mer tyrrhénienne. Outre cet itinéraire, il en existait deux autres: la route du détroit de Messine, avec circumnavigation de la péninsule calabraise, et la route qui traversait la Calabre au Sud-Ouest de la Basilicate, qui était contrôlée par Sybaris. En d'autres termes, l'intérêt pour les indigènes est surtout un intérêt porté à des gens qui disposent d'une filière de relations à longue distance.

Dans ce contexte, il est en outre très intéressant de noter que les deux partenaires, les Grecs et les Etrusques, commencent très tôt à laisser entre les mains de leurs interlocuteurs indigènes (ou, plutôt, des aristocraties indigènes évoquées ci-dessus), non plus des objets isolés, mais des ensembles d'objets liés à des systèmes concernant (dans le 
cadre d'une évolution générale des coutumes) deux domaines bien précis : celui des célébrations politiques et religieuses constituées autour de la consommation alimentaire des privilégiés et celui de l'organisation militaire.

21 L'importance du rôle de médiation joué par les Oenôtres pendant un siècle environ et cette évolution des échanges (qui désormais impliquent aussi le monde masculin) sont confirmées par les résultats des recherches conduites sur un deuxième site, Chiaromonte, situé dans la vallée voisine, celle du Sinni. Témoignant de l'acquisition d'objets provenant de l'un ou l'autre des deux versants, les tombes masculines du début $\mathrm{du}_{\mathrm{VI}}^{\mathrm{e}}$ siècle y montrent la complète adoption, aussi bien du rituel du banquet fondé sur les viandes rôties et sur le vin, que de l'armement hoplitique. La tombe au mobilier le plus somptueux qu'on ait trouvée jusqu'ici nous fournit d'une part un très grand « service » de cuisine et de table, en métal ou en céramique (y compris du bucchero), agrémenté de récipients à onguents et à parfums, et d'autre part un équipement d'hoplite à cheval, tel qu'on le voit représenté sur le fameux petit bronze italiote du British Museum, dit "Cavalier de Grumentum ", qui est à peine plus récent. Si l'on estime que cette pratique a pu avoir une signification plus large que la pure et simple acquisition d'un signe de status, elle pourrait suggérer l'hypothèse du début d'une forme d'agrégation de ces noyaux indigènes dans un plus large ensemble, seul en mesure de donner vie à des escadrons de cavalerie qui soient militairement fonctionnels.

L'évolution de l'Oenôtrie semble donc liée à l'histoire politique de Siris, et, après l'anéantissement de celle-ci, survenu au plus tard au début du $\mathrm{VI}^{\mathrm{e}}$ siècle av. J.-C., à celle de Sybaris, qui lui succède probablement aussi pour le contrôle de l'arrière-pays indigène. La chute brutale de Sybaris en 510 av. J.-C. déclenche une crise qui finit par toucher aussi ses partenaires de l'intérieur des terres: après les premières décennies $d u v^{\mathrm{e}}$ siècle, la documentation archéologique est en effet très rare dans tous les territoires de la Basilicate méridionale. A l'époque de la seconde bataille de Cumes (474 av. J.-C.), les vallées de l'Agri et du Sinni sont presque inhabitées.

A ce moment-là, le rôle d'intermédiaire à longue distance que jouait cette région est déjà fortement amoindri, sinon réduit à néant, par l'importance croissante d'un second itinéraire intérieur, plus septentrional, qui reliait deux autres cités achéennes jumelles : Métaponte et Poseidonia. Cet itinéraire suit le cours du Sélé (dans la plaine côtière duquel a été fondée la seconde de ces cités), puis celui de l'ofanto (fleuve qui coule vers la Mer adriatique, dont nous allons reparler), enfin ceux du Basento et du Bradano, qui se jettent au contraire dans la Mer ionienne, de part et d'autre du plateau peu élevé sur lequel était construite Métaponte. L'itinéraire traverse le territoire de peuples que nous ne sommes pas encore en mesure de nommer, et qui produisent les céramiques connues sous l'appellation de « série secondaire » du subgéométrique bichrôme. Ces peuples pratiquent l'inhumation en position recroquevillée, et montrent ainsi des affinités avec les Apuliens, qui sont leurs voisins. Par rapport à ceux qui les précédaient au cours du premier Age du Fer, ils marquent une certaine continuité.

24 L'établissement de Serra di Vaglio se trouve au centre de cet itinéraire aussi bien que des régions dont je viens de parler. Il représente en outre le niveau le plus élevé qu'ait atteint le processus d'hellénisation dans la mesogaia de l'Apennin ${ }^{1}$. On a trouvé là, datant des premières années $\mathrm{du} \mathrm{VI}$ siècle, une surface pavée (probablement un lieu de rencontre et d'échange), entourée de portiques couverts, que décore une frise continue en terre cuite estampée, avec un cortège de cavaliers (si l'on songe à ce que nous avons dit plus haut, il est sûr que le motif n'a pas été choisi au hasard), frise directement importée de 
Métaponte. A Métaponte, en effet, des plaques tout à fait analogues décorent aussi bien le plus ancien des temples urbains (celui d'Athéna, que l'on appelle temple $\mathrm{C}$ ) que le temple du sanctuaire extra-urbain de San Biagio alla Venella, dans la basse vallée du Basento.

Peu de temps après, à l'emplacement d'un village de cabanes qui se trouvait au voisinage, au sommet de la Serra, apparaît un habitat avec des constructions de plus en plus complexes. On utilise dans cet habitat des terres cuites architectoniques de type métapontin (bientôt imitées aussi sur place), et il se dote d'un embryon de plan d'urbanisme fondé sur la présence d'un axe prépondérant. Le long de cet axe, quelques édifices de grandes dimensions s'installent au cours du v $v^{e}$ siècle ; leur fonction, de type " palatial », ne peut pas ne pas rappeler les réalisations analogues du pays étrusque.

Dans le reste du territoire voisin, le mécanisme de croissance démographique et de structuration sociale, lié à l'établissement de rapports avec les peuples indigènes les plus évolués, que nous avons déjà décrit à propos des vallées méridionales, se produit à nouveau, et à peu près à partir du dernier quart du vile siècle av. J.-C. Il se poursuit tout au long du $\mathrm{vI}^{\mathrm{e}}$ siècle et pendant une bonne partie du siècle suivant.

Les signes tangibles de cette évolution paraissent avant tout limités au domaine des comportements sociaux et des biens de prestige. Ici, les élites locales, entre les mains desquelles se concentrent ces biens de prestige, ne cherchent pas (ou ne parviennent pas) à opérer des transformations structurelles telles que nous les avons décrites à Serra di Vaglio. Cela vaut même pour des sites très proches de Métaponte, comme Pisticci, où une récente découverte épigraphique de la fin de l'archaïsme amène pourtant à postuler la présence d'un noyau d'éphèbes envoyés faire le service militaire en tais eschatiais ${ }^{2}$.

En même temps que du matériel grec (il faut noter, après 440 av. J.C., les céramiques à figures rouges du groupe "protolucanien", dont au moins une partie est sûrement produite à Métaponte), les objets d'échange avec la Campanie continuent à être acheminés le long de cet itinéraire le plus intérieur, comme l'attestent avant tout les bronzes fondus de la fin de l'époque archaïque (dont certains semblent de fabrication étrusque plutôt qu'étrusco-campanienne) et quelques antéfixes caractéristiques, faciles à distinguer des antéfixes d'influence grecque.

C'est particulièrement net dans les sites de la partie Nord-Est de la Basilicate, qui gravite autour du Mont Vulture, et qu'on appelle aujourd'hui le Melfese, d'après le nom de la petite ville normande de Melfi.

A l'intersection des vallées de l'Ofanto et du Bradano, et à la limite de la plaine et de la montagne, cette partie Nord-Est constitue en effet une véritable "marche " entre les peuples dont nous venons d'évoquer les rapports avec les cités grecques et d'autre part les Dauniens (à l'Est) et les Hirpiniens (au Nord).

D'une façon beaucoup plus claire qu'ailleurs, se manifestent ici, sur un arrière-plan d'évidente contamination entre divers héritages culturels indigènes, des phénomènes plus poussés de structuration sociale et aussi d'acculturation. Si l'on en juge, comme toujours, par la documentation que fournit le rituel funéraire, cette zone semble avoir connu des conditions favorables à l'affirmation (dans le cadre de groupes gentilices) de dynastes et de petits rois (dynastai et reguli, pour employer le vocabulaire de l'historiographie grecque et latine). Séduits par les colliers et les ornements précieux (souvent de l'argent et des intailles d'ambre), en un mot par cette habrosyne, cette opulence raffinée de tradition ionienne qu'on a souvent reprochée aux Etrusques, ils étaient disposés à adopter des habitudes nouvelles telles que celle du symposion (à la place 
de l'ancien banquet), et à se rallier en outre à des comportements philosophico-religieux nouveaux et moins superficiels.

L'exemple le plus clair (mais qui n'est pas unique) est maintenant fourni par l'extraordinaire cas du mythe d'Eos et de son jeune aimé (Kephalos, Tithonos), métaphore bien connue du salut de l'âme grâce à l'intervention divine. Il figure en effet dans la tombe d'un couple, la plus riche et la plus récente qu'on ait trouvée à Ruvo del Monte (fin du $v^{e}$ siècle avant J.-C.) : dans la sépulture masculine, il est représenté sur le cratère, de fabrication métapontine; dans celle de la femme, à l'extrémité d'un candélabre de bronze fabriqué à Vulci.

du roi Daunus, mal pourvue en eau, dont parle Horace (pauper aquae Daunus, Odes $3,30,11)$, représente sans aucun doute un aspect tout à fait particulier du monde indigène, car la région où elle se trouve est longtemps demeurée la moins perméable aux influences culturelles grecques; c'est certainement la seule dont l'évolution n'ait pas été directement conditionnée par la colonisation grecque. Un apport fondamental à une meilleure connaissance du peuple daunien nous vient désormais des longues recherches consacrées à deux sites, actuellement situés à l'intérieur des limites de la Basilicate.

Le premier, Banzi (Bantia à l'époque antique), se trouve sur le rebord montagneux qui longe la haute vallée du Bradano, presque aux confins de la Peucétie. Le second, Lavello, proche de l'ofanto et dans l'arrière-pays de Canosa (Canusium), est le centre le plus important de la Daunie méridionale. Il est ouvert aux relations avec l'autre rive de l'Adriatique, vers laquelle il exporte en effet une catégorie particulière de céramique subgéométrique. Installé sur une vaste colline peu éloignée de la rive gauche du fleuve, le site de Lavello, très étendu et structuré, doit probablement être identifié avec ce Forentum... validum oppidum que les Romains enlèvent aux Samnites en 318-317 av. J.-C. (Tite-Live 9,20) ou en 315-314 av. J.-C. (Diodore de Sicile, 19,65,7).

Les fouilles attestent désormais comment les divers noyaux d'habitations, répartis sur toute la colline, prennent de l'ampleur au cours du viI siècle av. J.-C., et comment se manifestent à la même époque des signes d'une structuration sociale, avec le phénomène des tombes "princières ", qui sont du reste construites selon des schémas de composition assez éloignés de ceux que nous avons indiqués pour la Lucanie. Le mobilier des tombes qui correspondent à ces habitats montre en tout cas l'existence de rapports avec le milieu étrusque (par l'itinéraire Ofanto-Sélé, dont nous avons déjà parlé, et qui suppose aussi un rôle d'intermédiation de la part des Hirpiniens), et également, mais à un degré moindre, avec les colonies grecques de la côte ionienne, à travers la vallée du Bradano, ainsi sans doute qu'avec Siris.

Le mécanisme de croissance demeure actif au cours du siècle suivant, comme le montre avant tout le grand nombre des sépultures (dans lesquelles les importations helléniques, au moins tout au long $\mathrm{du} \mathrm{VI}^{\mathrm{e}}$ siècle av.J.-C. constituent un fait exceptionnel et quantitativement limité). Selon la coutume apulienne, ces sépultures flanquent chacun des noyaux d'habitations. Quant aux habitations, elles connaissent une évolution typologique mais très lente: on passe de cabanes, en général creusées dans le sol, à des structures dont l'élévation était construite en matériaux légers et qui adoptèrent une forme d'abord absidiale, puis rectangulaire.

Même dans ce cas, l'évolution ne s'accompagne cependant d'aucun processus de réorganisation d'ensemble de l'habitat, pour s'orienter vers des formes de synoecisme. On peut au contraire soupçonner que le renforcement de la structure per gentes, par 
groupements de parenté, explique la construction de deux ensembles distincts, de plan, de dimensions et d'orientation semblables. Ces deux ensembles sont très éloignés de tout prototype hellénique. Chacun d'eux est composé de deux secteurs distincts, l'un résidentiel (un groupe de plusieurs pièces), l'autre de fonction probablement " publique »: ce dernier est formé d'une longue pièce rectangulaire, tout au plus couverte d'un toit, et précédée par un ample vestibule, qui déborde de côté et d'autre. Ce sont très probablement les résidences de deux des groupes qui dominaient les noyaux d'habitations. Une confirmation est fournie par la proximité de quelques tombes contemporaines dignes d'être remarquées, tant par leur monumentalité architecturale que par le mobilier déplacé. Comme quelques autres qui ont été mises au jour en différents points de la colline et qui se rapportent donc à des situations analogues, elles présentent un mobilier en partie composé de formes céramiques qu'on ne trouve pas ailleurs (mais il y a aussi des importations attiques à figures rouges), de vaisselle métallique de provenance variée, souvent de notable qualité, et enfin d'éléments métalliques de l'équipement guerrier défensif.

La trouvaille d'un grand acrotère, qui représente, selon un schéma iconographique péloponnésien, le "Seigneur des chevaux " (il faut probablement songer aussi à un lien avec la tradition mythologique concernant la présence de Diomède en Daunie), témoigne, dans l'un de ces " palais ", d'un aspect particulier de la civilisation daunienne : l'intérêt porté à l'élevage des chevaux et à la cavalerie (Strabon, 6,3,9=C285), qui est attesté dès l'époque archaïque à travers des vases de cérémonie (donc au plan de l'imaginaire religieux) et est encore confirmé par la découverte d'une tombe de cheval.

D'autre part, à une époque qui semble postérieure au milieu du v siècle, un prolongement isolé du reste de la colline présente l'aspect d'une véritable acropole, dotée de fonctions et de structures spécifiques. A la place d'aménagements qui ne sont plus reconnaissables, est alors implanté un ensemble formé d'un petit oikos, situé dans l'alignement d'un enclos orienté, délimité par une série de fosses à offrandes (bothroi) : un cas unique dans l'état actuel de nos connaissances. Lors d'une réfection du second quart $\mathrm{du} \mathrm{IV}^{\mathrm{e}}$ siècle, les parois de cet oikos sont en partie ornées d'une frise à motifs décoratifs végétaux et géométriques, de type grec, obtenus en estampillant avec des tampons de bois une bande d'enduit encore frais. L'enclos voisin (dans lequel on a proposé de voir un templum augurale, lié au renouvellement des magistratures locales) conserve, dans divers petits puits, les restes d'une grande cérémonie d'inauguration (beaucoup de céramique fine de table) qui évoque les rituels ombriens des tables de bronze de Gubbio (tabulae igubinae).

$\mathrm{Au}$ cours de ces mêmes décennies, les tombes qui se répartissent dans cette zone prennent un aspect plus monumental (jusqu'à adopter le type de la tombe à chambre, construite en grand appareil avec couverture en fausse voûte, type attesté à la même époque en Thrace), suggèrent un rituel plus complexe et contiennent un mobilier bien plus riche que celles que nous avons précédemment décrites.

41 Les objets les plus marquants sont un groupe de petits vases en argent, de la céramique à figures rouges, d'abord importée d'Attique, puis apulienne, et surtout des armes de bronze, entre autres un casque de type chalcidien aux grandes aigrettes et un équipement guerrier de la fin du IV siècle av. J.-C. A la cuirasse pour cavalier, qui marque les formes anatomiques et au prometopidion correspondant pour le cheval, s'ajoute un casque à bouton sommital, provenant certainement d'Italie centrale, et probablement de Rome. 

renforcement d'un système "républicain » tel qu'on le connait dans le monde lucanien. Certains éléments rituels indiquent d'autre part qu'à leur mise en oeuvre ne sont sûrement pas étrangers des groupes samnites partis de leurs territoires de l'Apennin en Italie centrale. Après avoir établi des rapports à distance (probablement attestés par l'apparition, dans les sépultures de Basilicate, de typiques ceinturons à plaques rectangulaires de bronze), ces Samnites se sont ensuite directement infiltrés dans les régions de plaines et de collines qui bordent l'Adriatique, en une alternance d'affrontements et d'intégrations. Comme l'affirment les textes déjà cités, c'est à eux que les Romains enlèveront plus tard Forentum. plus marquée sur le versant opposé de l'Apennin et le long des plaines tyrrhéniennes, où, pour nous limiter au territoire situé au Sud du Sélé, elle atteint son plus grand succès avec la prise de Poseidonia ${ }^{3}$ vers $430-420$ av. J.-C.

Pour tout l'hellénisme occidental, cette prise de Poseidonia est un événement traumatisant. Il n'est d'ailleurs pas sans lien direct avec la crise qui, en Basilicate, frappe les régions intérieures tournées vers Métaponte, même si Métaponte elle-même a échappé, au cours de la première moitié de ce même siècle, à l'effondrement de Sybaris. Nous touchons ainsi au processus encore obscur, de l'ethnogénèse d'un nouveau groupement, celui des Lucaniens (destinés à donner par la suite leur nom à la région), groupement dont l'origine samnite est soulignée par tous les textes antiques. Notons à ce propos que les archéologues travaillant du côté de la Mer tyrrhénienne ont récemment insisté sur l'existence d'éléments de continuité par rapport aux populations indigènes précédentes.

Les deux faits suivants paraissent toutefois indubitables. D'une part, c'est seulement grâce à l'apport de la composante italique de l'intérieur qu'on arrive à la constitution d'une unité présentant des caractéristiques étatiques, parlant, au moins officiellement, une seule et unique langue, qui était aussi écrite, et désignée par un nom de peuple bien précis. D'autre part, ce processus d'ethnogénèse provoque la disparition presque totale de l'organisation territoriale précédente, à commencer par les sites de la région de Melfi dont nous avons décrit ci-dessus le développement : ils paraissent compter au nombre de ceux qui en ont le plus souffert.

Du reste, en analysant la répartition territoriale des différents cas qui se présentent tels qu'ils apparaissent désormais à partir d'un grand nombre de fouilles, il est facile d'arriver à la conclusion que les effets les plus négatifs concernent les sites du Nord et du Nord-Est, jusqu'à la limite de la Daunie intérieure. Dans les zones plus voisines du versant tyrrhénien, les transformations s'opèrent de façon moins explosive et n'interdisent pas une plus grande continuité.

47 On peut donc désormais formuler des hypothèses assez précises : c'est la fin du précédent réseau de relations entre les cités grecques qui a provoqué la crise d'une partie du vieux monde indigène. Comme l'indiquent toutes les manifestations culturelles et idéologiques, Poseidonia, une fois tombée entre les mains des Lucaniens, cesse en effet de servir de partenaire à Métaponte. La route de l'intérieur qui reposait sur leurs rapports, et sur laquelle s'est greffé et a prospéré le développement de ces peuples, perd à cette même époque toute sa raison d'être. Ce que nous avons vu se produire après la chute de Sybaris se répète donc, à environ un siècle de distance. 

nouvelle unité lucanienne, loin des côtes tyrrhéniennes, réponde à des critères complètement différents de ceux de l'époque archaïque, en rapport avec son homogénéité institutionnelle et culturelle, qui était destinée à durer au moins jusqu'à l'époque de Pyrrhus.

$49 \mathrm{Au}$ cours $\mathrm{du} \mathrm{IV}^{\mathrm{e}}$ siècle av. J.-C., la mesogaia entre la Mer tyrrhénienne et la Mer ionienne parait donc se refermer sur elle-même en une sorte d'autarcie. En l'absence totale de villes, ce qui n'est pas pour surprendre (elles n'apparaîtront qu'avec la domination romaine), on ne peut compter qu'un petit nombre de centres de dimensions importantes - par exemple, encore une fois, Serra di Vaglio, muni d'un puissant mur d'enceinte. Pour resserrer les mailles, trop larges sans cela, de ce système avant tout défensif, les communautés s'emploient officiellement (comme le prouve la mise en place d'inscriptions portant les noms des magistrats responsables) à construire sur les hauteurs un vaste réseau de sites fortifiés, tout à fait semblables à ceux qu'on connaît le long de la partie médiane de la chaîne de l'Apennin. Ces sites formaient une voie de rocade militaire et, en cas de nécessité, servaient de refuge pour les hommes et les biens.

conséquence, la population, vouée de manière absolument prédominante aux activités primaires, se répartit à travers tout le territoire en une trame serrée d'établissements, dont les dimensions sont le plus souvent celles de simples fermes ou de hameaux ruraux, et que presque toujours nous ne connaissons qu'à travers les petites nécropoles correspondantes. Le mobilier funéraire renvoie une image de l'idéologie qui caractérise ces petits groupes patriarcaux, idéologie plus simple et transparente que celle des cultures archaïques (ou même que celle des Apuliens voisins). Parallèlement aux deux pôles sexuels qui représentent les armes (lances de fer et ceinturons de bronze) et un petit nombre d'objets de toilette, il y a la présence constante (une présence qui n'est souvent que symbolique) d'instruments utilisés au foyer domestique, centre de la vie de chacun des membres du genos.

51 A une occupation du territoire aussi dispersée répondent de remarquables possibilités militaires, comme nous le font savoir les textes historiques grecs et latins. Cela révèle, sans aucun doute possible, l'existence d'une organisation politique et administrative efficace qui nécessitait à son tour l'existence de liaisons matérielles. Les sanctuaires, indépendants des habitats (selon un modèle qui rappelle là encore ceux d'Italie centrale) et situés dans des lieux topographiquement marquants, semblent avoir été appelés à jouer ce rôle de lieu de rencontre et d'échange. L'ampleur des constructions qu'on y trouve et la richesse des ex votos qui y sont offerts montrent l'intérêt qu'éprouvait à leur égard la communauté. On a du reste démontré récemment qu'au cours de la période républicaine romaine qui a suivi, le plus imposant et le plus central de ces lieux de culte, le sanctuaire de Mefite construit à Rossano di Vaglio, qui se caractérise par une évidente continuité, est probablement impliqué (comme le montre l'épithète Utiana attribuée à la déesse) dans des rapports de patronage avec les Utiani, une des gentes les plus importantes de la Lucanie, qui possèdent à Polla un grand monument funéraire. militaire), aussi bien que dans le domaine des formes d'expression, les Lucaniens ont manifestement contracté une dette à l'égard des techniques grecques. Mais dans leurs prises de position fondamentales, dans le choix des paramètres qui guident leur vie, ils apparaissent désormais comme très éloignés des Italiques d'époque archaïque. A la différence de ceux-ci, ils ont en effet cessé de dépendre structurellement des Italiotes. Au 
contraire, ils en assimilent la culture et les techniques, en fonction d'un évident antagonisme politique, que n'atténuent pas même des phénomènes aussi marquants au plan idéologique que l'adhésion à la doctrine pythagoricienne de la part des porte-parole de leur aristocratie.

En 389 av. J.-C., les Grecs de Thourioi essaient de pénétrer manu militari dans les hauteurs de l'Apennin lucanien, et leur tentative se solde par la mémorable défaite de Laos : ces événements de 389 av. J.-C. symbolisent la nouvelle situation de cette région ${ }^{4}$.

\section{Bibliographie}

\section{Limites de la région}

R. THOMSEN, The Italic regions from Augustus to the Lombard invasion, Copenhague, 1947, p. 79 sq.

\section{Synthèses historiques et étude des sources textuelles}

\section{Synthèses archéologiques}




\section{Musées}

71

\section{NOTES}

1. Mesogaia signifie l'intérieur des terres (N.d.T.)

2. Aux frontières de la cité, à la périphérie de son territoire (N.d.T.)

3. La colonie grecque de Poseidonia prend par la suite le nom de Paestum (N.d.T.)

4. Nous remercions vivement M. GRAS, qui a eu la gentillesse de revoir la traduction de cet article (N.d.T.)

\section{AUTEUR}

\section{ANGELO BOTTINI}

Angelo BOTTINI est directeur des Antiquités archéologiques de Basilicate.The Basilicata is not an attractive or fertile country, though it was perhaps less barren and treeless in Antiquity. It is now one of the poorest regions of Italy. The Greeks very reasonably stayed in their rich plain and left the windy barren mountains to their own people. 